\title{
Hearing Improvement in A/J Mice via the Mouse Nerve Growth Factor
}

\author{
Lixiang Gao ${ }^{1,2} \cdot$ Ruli Ge ${ }^{1,2} \cdot$ Gang Xie ${ }^{1}$ Dandan Yao $\cdot$ Ping $\mathrm{Li}^{1} \cdot$ Oumei Wang ${ }^{1}$ Xiufang Ma1,3 Fengchan Han,4 \\ ${ }^{1}$ Key Laboratory for Genetic Hearing Disorders in Shandong, Binzhou Medical University, Yantai; \\ Departments of ${ }^{2}$ Neurology and ${ }^{3}$ Otorhinolaryngology Head and Neck Surgery, University Hospital of Binzhou Medical University, Binzhou; \\ ${ }^{4}$ Department of Biochemistry and Molecular Biology, Binzhou Medical University, Yantai, China
}

Objectives. To investigate the otoprotective effects of mouse nerve growth factor (mNGF) in A/J mice.

Methods. The mice at postnatal day 7 (P7) were randomly separated into a mNGF treated group (mNGF group) and a distilled water (for injection) treated group (control group). The mNGF dissolved in distilled water or distilled water alone was given to the mice once every other day from P7 by intramuscular injection in the hips. The otoprotective effects of $\mathrm{mNGF}$ in $\mathrm{A} / \mathrm{J}$ mice were observed in a time course manner. The thresholds of auditory-evoked brainstem response (ABR) were tested from the age of the 3rd to the 8th week. Sections of the inner ears were stained by hematoxylin and eosin, and spiral ganglion neurons (SGNs) were observed at the age of the 3rd, the 6th, and the 8th week. Counts of whole mount outer hair cells (OHCs) in the cochleae were made at the age of 8 weeks. Expression of apoptosis related genes was determined by quantitative real-time polymerase chain reaction and Western blotting.

Results. ABR thresholds of the mNGF group were significantly lower than those of the control group at the age of the 6th and the 8th week. Moreover, the mNGF preserved OHC and SGN in the mouse cochleae in this period. Further experiments showed that the expression of caspase genes (including caspase-3) was inhibited in the mouse inner ears in the mNGF group.

Conclusion. The mNGF improves hearing in A/J mice by preserving SGN and OHC in the cochleae.

Keywords. Nerve Growth Factor; Hair Cells; Neurons; Protection

\section{INTRODUCTION}

Hearing loss in $\mathrm{A} / \mathrm{J}$ mice begins at the age of about 1 month [1]. Studies have revealed that mutations in genes of cadherin 23

\footnotetext{
- Received October 2, 2016

Revised December 26, 2016

Accepted January 19, 2017

- Corresponding author: Fengchan Han

Key Laboratory for Genetic Hearing Disorders in Shandong, Binzhou

Medical University, 346 Guanhai Road, Yantai 264003, China

Tel: +86-535-691-3480, Fax: +86-535-691-3163

E-mail: hanfengchan@gmail.com

- Co-Corresponding author: Xiufang Ma

Department of Otorhinolaryngology Head and Neck Surgery, University

Hospital, Binzhou Medical University, 661 Second Huanghe Road, Binzhou 256603, China

Tel: +86-543-325-8739, Fax: +86-543-325-8850

E-mail: drmxf1969@163.com
}

(ahl) [2,3], citrate synthase (ahl4) [4], and tRNA-Arg (mt-tr) [5] contribute to the early onset of hearing loss. Histologically, the mice display progressive loss of hair cells and spiral ganglion neurons (SGNs) [6]. Recent studies show that caspase-mediated apoptosis is involved in the cochlear pathology [7]. These characteristics provide possibilities for drug intervention in the hearing impairment.

Nerve growth factor (NGF) is essential for viability, differentiation, and maintenance of nerve cells [8]. It belongs to a superfamily of neurotrophins, which also includes the brain-derived neurotrophic factor (BDNF), the neurotrophin-3 (NT-3), and others. Actually, NGF, BDNF, and NT-3 function by being combined with the tyrosine kinase receptor A (TrkA), TrkB, and TrkC, respectively [9]. Among them, NGF is the first one used to prevent auditory nerve (neuron or fiber) degeneration caused by neomycin in guinea pigs [10]. NGF also stimulates neurite

Copyright @ 2017 by Korean Society of Otorhinolaryngology-Head and Neck Surgery.

This is an open-access article distributed under the terms of the Creative Commons Attribution Non-Commercial License (http://creativecommons.org/licenses/by-nc/4.0)

which permits unrestricted non-commercial use, distribution, and reproduction in any medium, provided the original work is properly cited. 
outgrowth from implanted neurons in the adult rat inner ears [11] and improves the survival of statoacoustic ganglion-derived neural progenitors in vitro and in vivo [12]. Moreover, clinical studies have revealed that NGF level in serum is relatively lower in patients with sensorineural hearing defects [13].

Therefore, the otoprotective effects of mNGF in A/J mice with genetic hearing loss have been observed in the present study. Our findings are that mNGF can improve hearing in A/J mice and preserve outer hair cells (OHCs) and SGN in the cochleae.

\section{MATERIALS AND METHODS}

\section{Mouse preparations and treatments}

$\mathrm{A} / \mathrm{J}$ mice were bred in a specific pathogen free facility. A total of 184 mice (98 males and 86 females) with ages ranging from postnatal day 7 (P7) to P60 were included in this study. The methods and procedures of animal studies were approved by the Animal Use and Care Committee of Binzhou Medical University. Mouse pups at P7 were randomly separated into a mNGF treated group (mNGF group) and a distilled water (for injection) treated group (control group). The mNGF was purified from mouse submaxillary gland (Catalog No. 20131213; Sinobioway Biomedicine Co., Xiamen, China) and dissolved in distilled water at a concentration of $9 \times 10^{-3} \mu \mathrm{g} / \mu \mathrm{L}$. It was given to the mice in the mNGF group by intramuscular injection in the hips at a dosage of $3.6 \times 10^{-3} \mu \mathrm{g} / \mathrm{g}$ body mass once every other day. The dosages were calculated according to the methods previously described [14]. The mice in the control group received an equivalent amount of distilled water ( $0.4 \mu \mathrm{L} / \mathrm{g}$ body mass).

\section{Measurement of mouse ABR (auditory-evoked brainstem re- sponse) thresholds}

Auditory-evoked brainstem response (ABR) thresholds were tested in accord with the methods described previously $[15,16]$. The mice at the age of the 3rd, the 4th, the 6th, and the 8th week in the control group ( $\mathrm{n}=14$ for all the time points) and mNGF group ( $\mathrm{n}=12$ for all the time points) were subjected to the measurement.

\section{H I G G H L I G G H T S}

- Mouse nerve growth factor (mNGF) treatment attenuated hearing loss of experimental mice.

- Outer hair cell and spiral ganglion neuron were preserved by the treatment.

- The treatment inhibited caspase-3 expression in the inner ears of mice.

- mNGF has the protective effects of the cochleae.

\section{Surface preparations for hair cell counting}

The mice in the mNGF and control groups at the age of 8 weeks were used for evaluating hair cell preservation $(n=4$ in each group). The organ of Corti was microdissected out and mounted in glycerin on glass slides. The surface preparations were stained for F-actin with Alexa Fluor 488 phalloidin (1:500 dilution) and observed with a confocal fluorescence microscope (Leica DM4000 B, Leica Microsystems, Wetzlar, Germany) [16]. OHC was counted as present if $\mathrm{V}$-shape of hair bundles was intact. Counts of OHC in three continuous views for each turn of a cochlea were recorded and analysed [17].

\section{Histological analysis of SGN in the cochlea}

Five mice in the mNGF group and five in the control group at the age of the 3rd, the 6th, and the 8th week were randomly chosen and anaesthetised with avertin $(0.5 \mathrm{mg} / \mathrm{g}$ body mass). Sections of the inner ears were prepared, and SGN was observed and counted in accord with the methods described previously $[7,15]$.

\section{Detection of gene expression levels}

Quantitative polymerase chain reaction (PCR) was carried out for transcription levels of apoptosis related genes in the mice of mNGF group and control group at the age of the second, the fourth and the 8th week ( $n=5$ or 6 at each time point for both groups). RNA extraction, cDNA preparation and quantitative PCR were performed with the methods previously described $[7,15,18]$. The primers were listed in Table 1 and synthesised by Sangon Biotech Co. (Shanghai, China). The cleaved caspase-3 protein levels were also detected by Western blotting [7]. Four mice at the age of 8 weeks from each group were included.

\section{Statistical analysis}

ANOVA (SPSS ver. 16; SPSS Inc., Chicago, IL, USA) was used to analyse the data. Chi-square test was applied to analyse the percentage of OHC loss. $P$-value less than 0.05 was considered as significant.

Table 1. Primers for real-time polymerase chain reaction

\begin{tabular}{llc}
\hline ID & \multicolumn{1}{c}{ Sequence } & $\begin{array}{c}\text { Product size } \\
(\mathrm{bp})\end{array}$ \\
\hline Gapdh-F & 5'-CTTCCGTGTCCTACCCCCAATGT-3' & 100 \\
Gapdh-R & 5'-GCCTGCTTCACCACCTCTTGATG-3' & \\
Caspase-3F & 5'-TGTCATCTCGCTCTGGTACG-3' & 201 \\
Caspase-3R & 5'-AAATGACCCCTTCATCACCA-3' & \\
Caspase-9F & 5'-CCTAGTGAGCGAGCTGCAAG-3' & 232 \\
Caspase-9R & 5'-ACCGCTTGCAAGAGTGAAG-3' & \\
Caspase-12F & 5'-GCCCATGTGGAGACAGATT-3' & 206 \\
Caspase-12R & 5'-ATAGTGGGCATCTGGGTCAG-3' & \\
Bak-F & 5'-TGAAAAATGGCATCTGGACA-3' & 136 \\
Bak-R & 5'-CCTGCTGGTGGAGGTAAAAA-3' & \\
Bax-F & 5'-TGTTGCTGATGGCAACTTC-3' & 206 \\
Bax-R & 5'-GGTCCCGAAGTAGGAGAGGA-3' & \\
\hline
\end{tabular}



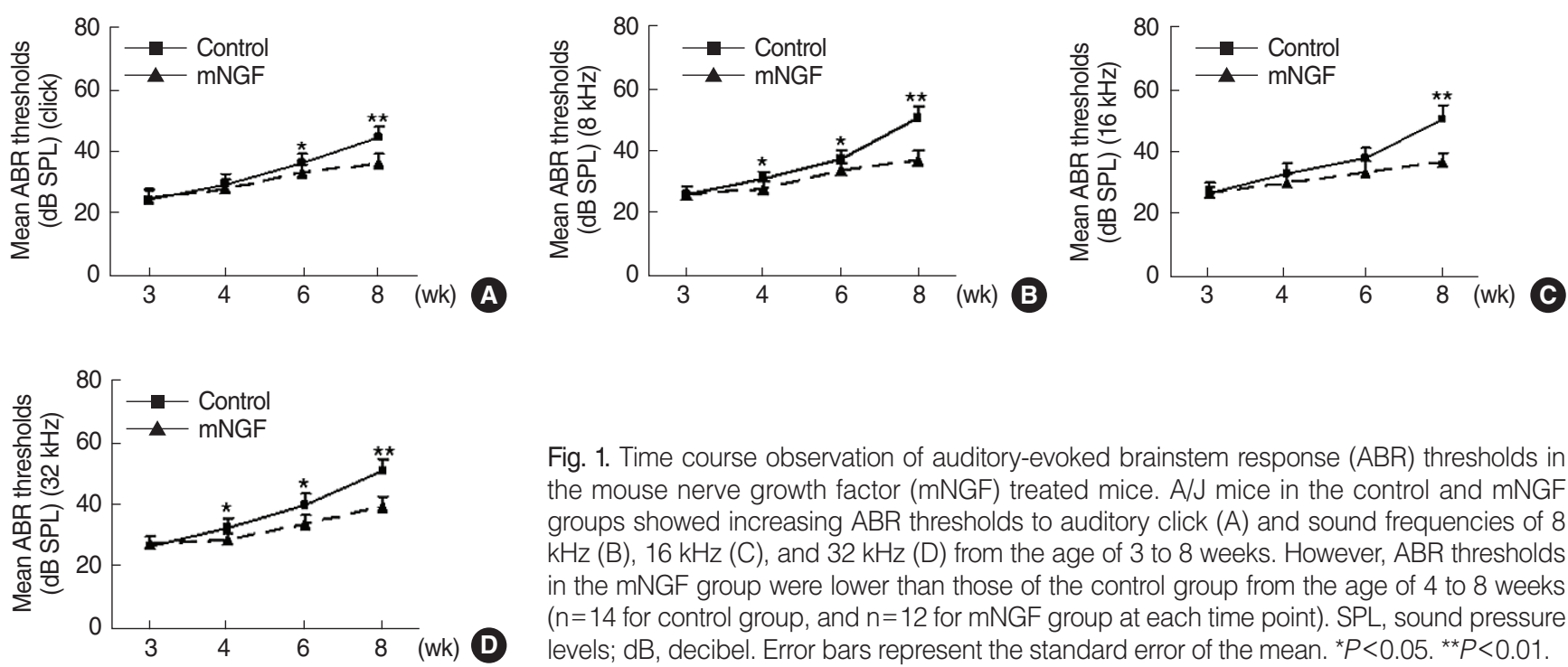

Fig. 1. Time course observation of auditory-evoked brainstem response (ABR) thresholds in the mouse nerve growth factor (mNGF) treated mice. A/J mice in the control and mNGF groups showed increasing ABR thresholds to auditory click $(A)$ and sound frequencies of 8 $\mathrm{kHz}(\mathrm{B}), 16 \mathrm{kHz}(\mathrm{C})$, and $32 \mathrm{kHz}$ (D) from the age of 3 to 8 weeks. However, ABR thresholds in the mNGF group were lower than those of the control group from the age of 4 to 8 weeks ( $n=14$ for control group, and $n=12$ for mNGF group at each time point). SPL, sound pressure levels; $\mathrm{dB}$, decibel. Error bars represent the standard error of the mean. ${ }^{*} P<0.05 .{ }^{*} P<0.01$.
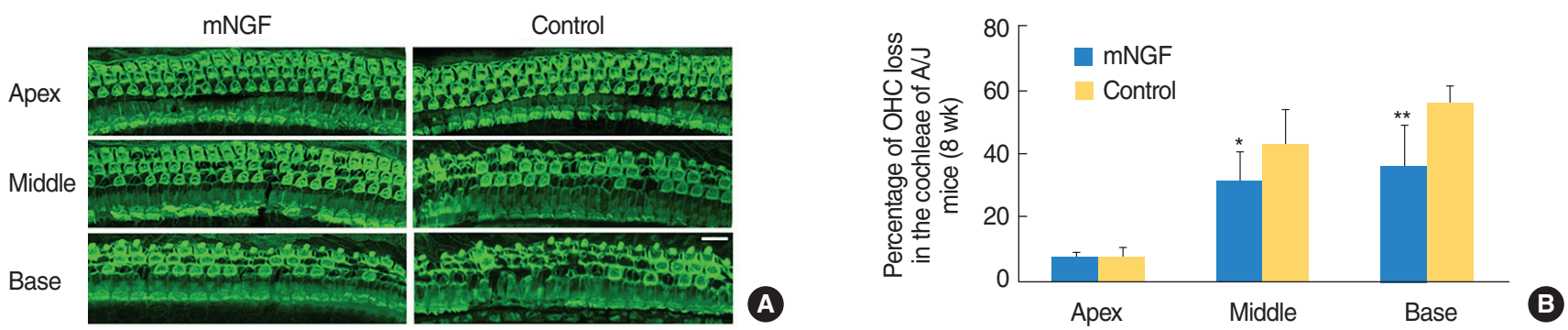

Fig. 2. Preservation of outer hair cell $(\mathrm{OHC})$ in the cochleae of mice in mouse nerve growth factor (mNGF) group. Hair cells were stained for Factin with Alexa Fluor 488-labeled phalloidin. (A) Representative OHC images in the three turns of mNGF and control mice at the age of 8 weeks. OHC loss at middle turns or basal turns of the control group was more severe than that of the $\mathrm{mNGF}$ group (scale bar=50 $\mu \mathrm{m})$. (B) Percentages of $\mathrm{OHC}$ loss in the three cochlear turns of both mouse groups at the age of 8 weeks. Percentage of $\mathrm{OHC}$ loss at middle turns or basal turns in mNGF group was significantly lower than that in the control group. There was only a small number of $\mathrm{OHC}$ loss at the apical turns in both mouse groups ( $\mathrm{n}=4$ for each group). Error bars represent the standard error of the mean. ${ }^{*} P<0.05$. ${ }^{* *} P<0.01$.

\section{RESULTS}

\section{Hearing improvement in the mNGF treated mice}

ABR thresholds of the two mouse groups were measured and were shown in Fig. 1. mNGF significantly decreased the ABR thresholds to auditory click and pure tone stimuli $(8,16$, and 32 $\mathrm{kHz})$ in mice at the age of 8 weeks $(P<0.001$, respectively). mNGF also significantly lowered the ABR thresholds to click and certain pure tone stimuli at the age of 6 weeks $(P=0.032$, $P=0.046, P=0.068$, and $P=0.007$, respectively, for click, 8,16 , and $32 \mathrm{kHz})$ and 4 weeks $(P=0.468, P=0.048, P=0.050$, and $P=0.012$, respectively). mNGF showed no significant effects on the mouse hearing at the age of 3 weeks $(P=0.775, P=0.944$, $P=0.929$, and $P=0.509$, respectively). However, ABR thresholds in the mNGF group still increased with age during this period.

\section{$\mathrm{OHC}$ protection in the mNGF treated mice}

Typical images of $\mathrm{OHC}$ distribution at the three turns of cochle- ae of the mNGF and control groups at the age of 8 weeks were shown in Fig. 2A, and percentage of OHC loss in each turn of each group was displayed in Fig. 2B. Actually, there was about $7 \%$ OHC loss at the apical turns in the cochleae of both mouse groups. The rates of $\mathrm{OHC}$ loss were $31 \%$ and $35 \%$, respectively, at the middle and basal turns in the mNGF group, whereas the percentages were $42 \%$ and $55 \%$ at the same areas in the control mice. OHC loss was significantly less in the mNGF group than that in the control group at middle turns $(P<0.01)$ or basal turns $(P<0.01)$.

\section{SGN preservation in the cochleae of mNGF treated mice}

The densities of SGN at the three cochlear turns were observed in both mouse groups at the age of the 3rd, the 6th, and the 8th week. The results revealed that mNGF had no significant effects $(P>0.05)$ on SGN densities at any turn of mice at the age of 3 weeks. However, SGN densities at apical, middle, and basal turns of mice in the mNGF group were significantly higher than 

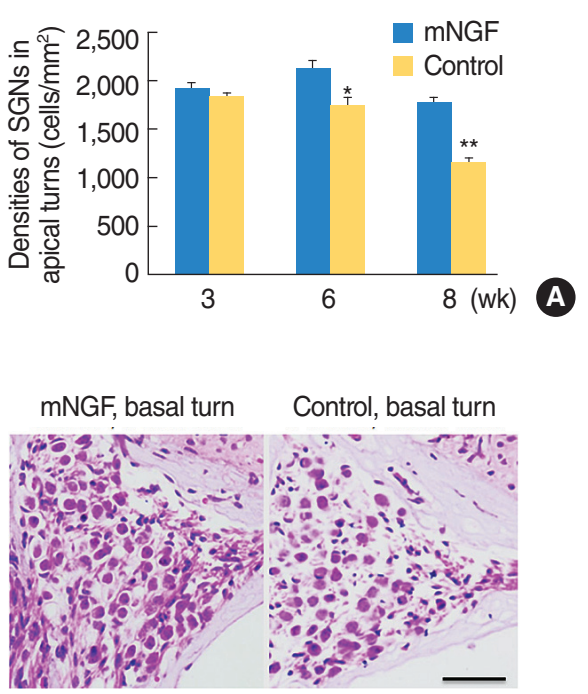

8 Weeks

8 Weeks
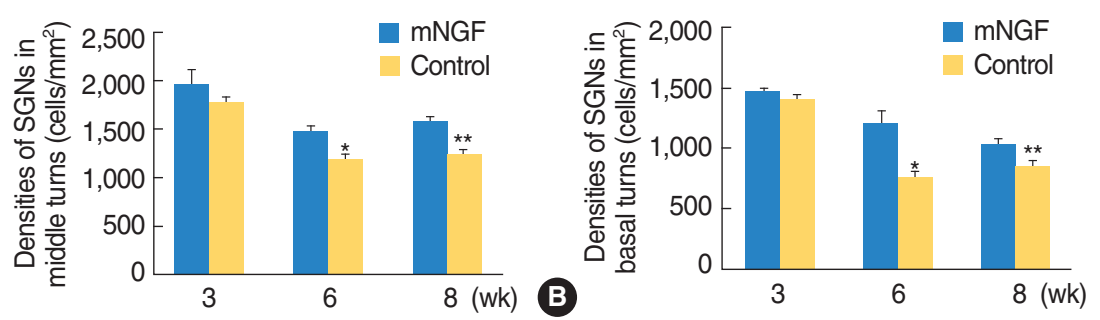

Fig. 3. Preservation of spiral ganglion neurons (SGNs) in the mice of mouse nerve growth factor (mNGF) group. (A-C) Densities of SGN at the three cochlear turns in the mNGF and control groups. At the age of 3 weeks, there were no significant differences of SGN densities between the two mouse groups at any turn $(P>0.05)$. Whereas the densities in $\mathrm{mNGF}$ group were significantly higher than those in the control group at the age of 6 weeks $\left({ }^{\star} P=\right.$ $0.021, P=0.032$, and $P=0.035$, respectively, between apical, middle, and basal turns) and 8 weeks $\left({ }^{\star \star} P=0.010, P=0.021\right.$, and $P=0.023$, respectively, between apical, middle, and basal turns) ( $n=5$ for each group at each time point). Error bars represent the standard error of the mean. (D) Representative images of SGN at basal turns of the mNGF and control groups at the age of 8 weeks. The control mice showed relative sparse SGN, which proved that $\mathrm{mNGF}$ preserved SGN in A/J mice. Scale bar $=50 \mu \mathrm{m}$.
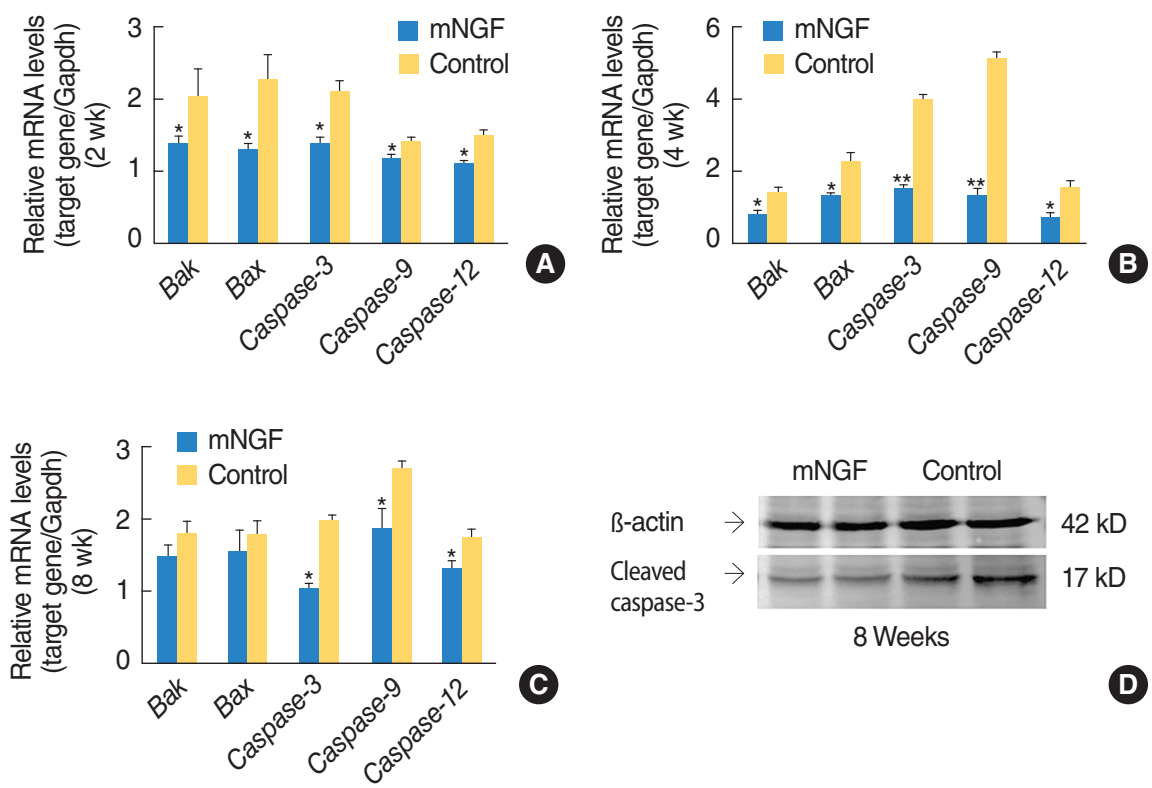

Fig. 4. Gene expression profiles in the inner ears of mice in the mouse nerve growth factor (mNGF) group. mRNA levels of Bak, Bax, Caspase-3, Caspase-9, and Caspase-12 in the inner ears of the mNGF group were lower than those of the control group, with significant differences of the 5 genes at the age of 2 weeks ( $n=6$ for each group) (A), 4 weeks ( $n=6$ for each group) (B), and with significant differences of Caspase-3, Caspase-9, and Caspase-12 at the age of 8 weeks ( $n=5$ for each group) (C).

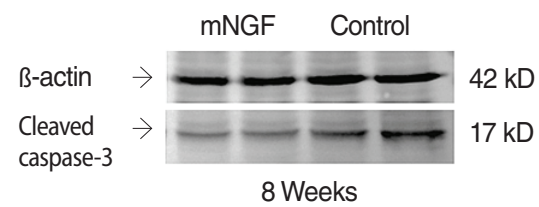
Error bars represent the standard error of the mean. ${ }^{*} P<0.05$. ${ }^{*} P<0.01$. (D) Western blotting images of the cleaved caspase-3 $(17 \mathrm{kD})$ in the inner ears of the two mouse groups at the age of 8 weeks. The results showed that the levels of cleaved caspase-3 in the mouse inner ears of mNGF group were downregulated compared with the levels of the control group. the densities of the control group at the age of 6 weeks $(P=$ $0.021, P=0.032$, and $P=0.035$, respectively) and 8 weeks $(P=$ 0.010, 0.021, and 0.023, respectively) (Fig. 3A-C). Representative images of SGN in the basal turns of mice at the age of 8 weeks were shown in Fig. 3D.

\section{Inhibiting apoptosis related gene expression in the mouse in- ner ears by $\mathrm{mNGF}$}

Gene expression profiles of apoptosis related pathways were detected in the inner ears of mice at the age of the 2nd, the 4th, and the 8th week. Transcription levels of Bak, Bax, Caspase-9, Caspase-12, and Caspase-3 in the mNGF group were overall lower than the levels in the control group at the age of 2 weeks
$(P=0.024, P=0.023, P=0.013, P=0.031$, and $P=0.001$, respectively), 4 weeks $(P=0.016, P=0.018, P=0.002, P=0.000$, and $P=0.012$, respectively), and 8 weeks $(P=0.235, P=0.558, P=$ $0.013, P=0.028$, and $P=0.014$, respectively) (Fig. $4 \mathrm{~A}-\mathrm{C}$ ). Decline in Caspase mRNA levels in the mNGF treated mice was most remarkable at the age of 4 weeks. Downregulation of cleaved caspase- 3 protein in the mice of $\mathrm{mNGF}$ group at the age of 8 weeks was further confirmed by Western blotting (Fig. 4D). However, mRNA levels of Caspase- 8 were not significantly different between the two mouse groups at any of the three time points (data not shown). Thus the above results indicated that Bak-mediated apoptotic pathway might be involved in hearing improvement in the mNGF treated mice. 


\section{DISCUSSION}

In this study, we observed the otoprotective effects of mNGF on a mouse model of genetic hearing loss. We first showed that mNGF attenuated the hearing impairment of A/J mice. Further experiments demonstrated that both $\mathrm{OHC}$ and SGN were preserved in the cochleae of mNGF treated mice. Thus, we concluded that $\mathrm{mNGF}$ might improve hearing in $\mathrm{A} / \mathrm{J}$ mice by protecting $\mathrm{OHC}$ and SGN against loss.

Early studies showed that TrkA was not essential for development of the avian peripheral auditory system [19], nor for development of mouse inner ears [20]. However, later studies revealed that the presence of $\operatorname{Trk} A, \operatorname{TrkB}$, and $\operatorname{Trk} C$ in the cochleae of adult guinea pigs, and BDNF, NT-3 or NGF alone could prevent SGN degeneration [21], indicating that SGN might respond to all the neurotrophins for survival. Moreover, the experiments in mice showed that TrkA receptor existed in hair cells at as early as postnatal day 6, and expressed in hair cells, supporting cells and SGN in the cochleae of the adult [22]. The patterns of TrkA distribution suggested that NGF had specific functions in the peripheral auditory system.

Actually, NGF can bind to the high-affinity receptor TrkA and a low-affinity neurotrophin receptor called p75NTR [23,24]. Physiological levels of NGF in the blood were considered to bind with TrkA in the cochleae and to exert its roles in sensory hearing impairment in humans [13]. When binding with TrkA, NGF becomes phosphorylated and acts as a binding site for specific signaling protein such as phosphatidylinositol 3-kinase (PI3K)/Akt, which phosphorylates Bad (BCL2-associated agonist of cell death), leading to inhibition of apoptosis pathway [25]. Therefore, the exogenous mNGF in this study might enter through the blood to the cochleae and bind with $\operatorname{TrkA}$ and/or p75NTR, exerting its otoprotective effects.

Our experiments also showed that expression of apoptosis related genes, Bak, Bax, and caspase-3, was inhibited in the mouse inner ears of $\mathrm{mNGF}$ group. These results suggested that the NGF-associated pathways were probably activated after the treatment, resulting in inhibition of caspase-3. As previous studies have already shown that the cleaved caspase- 3 mainly exists in OHC, SGN and stria vascularis of the cochleae [7], inhibition of caspase- 3 by mNGF in the inner ears may indicate downregulation of caspase-3 expression in OHC and SGN. The mechanism may be that $\mathrm{mNGF}$ protects $\mathrm{OHC}$ and SGN in the cochleae by inhibiting apoptotic pathways. However, the exact NGFassociated pathways in the cochleae remain unclear and need further investigation.

In conclusion, hearing impairment in $\mathrm{A} / \mathrm{J}$ mice is attenuated by mNGF. The effects may be obtained by preserving SGN and $\mathrm{OHC}$ in the cochleae. Inhibition of apoptosis related pathways is likely involved in the otoprotection. This is the first report that mNGF has been shown to have otoprotective effects on a mouse model of age-related hearing loss.

\section{CONFLICT OF INTEREST}

No potential conflict of interest relevant to this article was reported.

\section{ACKNOWLEDGMENTS}

This study was supported by grants of the National Natural Science Foundation (No. 81271092, 81570927) funded by the Ministry of Science and Technology, China, and grants of Scientific and Technological Development Foundation (No. 2014 GSF118083) and Natural Science Foundation (No. ZR2012 HL31) funded by the Bureau of Science and Technology, Shandong, China.

\section{REFERENCES}

1. Zheng QY, Ding D, Yu H, Salvi RJ, Johnson KR. A locus on distal chromosome 10 (ahl4) affecting age-related hearing loss in A/J mice. Neurobiol Aging. 2009 Oct;30(10):1693-705.

2. Johnson KR, Erway LC, Cook SA, Willott JF, Zheng QY. A major gene affecting age-related hearing loss in C57BL/6J mice. Hear Res. 1997 Dec;114(1-2):83-92.

3. Johnson KR, Zheng QY, Erway LC.A major gene affecting age-related hearing loss is common to at least ten inbred strains of mice. Genomics. 2000 Dec;70(2):171-80.

4. Johnson KR, Gagnon LH, Longo-Guess C, Kane KL. Association of a citrate synthase missense mutation with age-related hearing loss in A/J mice. Neurobiol Aging. 2012 Aug;33(8):1720-9.

5. Johnson KR, Zheng QY, Bykhovskaya Y, Spirina O, Fischel-Ghodsian N. A nuclear-mitochondrial DNA interaction affecting hearing impairment in mice. Nat Genet. 2001 Feb;27(2):191-4.

6. YamasobaT, Someya S, Yamada C, Weindruch R, Prolla TA, Tanokura M. Role of mitochondrial dysfunction and mitochondrial DNA mutations in age-related hearing loss. Hear Res. 2007 Apr;226(1-2):18593.

7. Han X, Ge R, Xie G, Li P, Zhao X, Gao L, et al. Caspase-mediated apoptosis in the cochleae contributes to the early onset of hearing loss in A/J mice. ASN Neuro. 2015 Jan-Feb;7(1):1-13. https://doi. org/10.1177/1759091415573985.

8. Bibel M, Barde YA. Neurotrophins: key regulators of cell fate and cell shape in the vertebrate nervous system. Genes Dev. 2000 Dec; 14(23):2919-37.

9. Neet KE, Campenot RB. Receptor binding, internalization, and retrograde transport of neurotrophic factors. Cell Mol Life Sci. 2001 Jul;58(8):1021-35.

10. Shah SB, Gladstone HB, Williams H, Hradek GT, Schindler RA. An extended study: protective effects of nerve growth factor in neomycin-induced auditory neural degeneration. Am J Otol. 1995 May;16 (3):310-4.

11. Hu Z, Ulfendahl M, Olivius NP. NGF stimulates extensive neurite outgrowth from implanted dorsal root ganglion neurons following transplantation into the adult rat inner ear. Neurobiol Dis. 2005 Feb; 18(1):184-92

12. Zhang L, Jiang $\mathrm{H}, \mathrm{Hu} \mathrm{Z}$. Concentration-dependent effect of nerve growth factor on cell fate determination of neural progenitors. Stem Cells Dev. 2011 Oct;20(10):1723-31. 
13. Salvinelli F, Casale M, Greco F, Trivelli M, Di Peco V,Amendola T, et al. Nerve growth factor serum level is reduced in patients with sensorineural hearing impairment: possible clinical implications. J Biol Regul Homeost Agents. 2002 Jul-Sep;16(3):176-80.

14. Reagan-Shaw S, Nihal M, Ahmad N. Dose translation from animal to human studies revisited. FASEB J. 2008 Mar;22(3):659-61.

15. Yang L, Zhang H, Han X, Zhao X, Hu F, Li P, et al. Attenuation of hearing loss in DBA/2J mice by anti-apoptotic treatment. Hear Res. 2015 Sep;327:109-16.

16. Han F, Yu H, Tian C, Chen HE, Benedict-Alderfer C, Zheng Y, et al. A new mouse mutant of the Cdh23 gene with early-onset hearing loss facilitates evaluation of otoprotection drugs. Pharmacogenomics J. 2012 Feb;12(1):30-44.

17. Hu J, Xu M, Yuan J, Li B, Entenman S, Yu H, et al. Tauroursodeoxycholic acid prevents hearing loss and hair cell death in Cdh23(erl/ erl) mice. Neuroscience. 2016 Mar;316:311-20.

18. Bookout AL, Mangelsdorf DJ. Quantitative real-time PCR protocol for analysis of nuclear receptor signaling pathways. Nucl Recept Signal. 2003;1:e012.

19. Pirvola U, Hallbook F, Xing-Qun L, Virkkala J, Saarma M, Ylikoski J.
Expression of neurotrophins and Trk receptors in the developing, adult, and regenerating avian cochlea. J Neurobiol. 1997 Dec;33(7): 1019-33.

20. Schimmang T, Alvarez-Bolado G, Minichiello L, Vazquez E, Giraldez F, Klein R, et al. Survival of inner ear sensory neurons in trk mutant mice. Mech Dev. 1997 Jun;64(1-2):77-85.

21. Gillespie LN, Clark GM, Marzella PL. Delayed neurotrophin treatment supports auditory neuron survival in deaf guinea pigs. Neuroreport. 2004 May;15(7):1121-5.

22. Dai CF, Steyger PS, Wang ZM, Vass Z, Nuttall AL. Expression of Trk A receptors in the mammalian inner ear. Hear Res. 2004 Jan;187(12):1-11.

23. Yano H, Chao MV. Neurotrophin receptor structure and interactions. Pharm Acta Helv. 2000 Mar;74(2-3):253-60.

24. Gillespie LN, Shepherd RK. Clinical application of neurotrophic factors: the potential for primary auditory neuron protection. Eur J Neurosci. 2005 Nov;22(9):2123-33.

25. Lee YR, Hong BN, Her YR, Castaneda R, Moon HW, Kang TH.Amelioration of auditory response by DA9801 in diabetic mouse. Evid Based Complement Alternat Med. 2015;2015:230747. 